Article

\title{
Perturbation of One-Dimensional Time Independent Schrödinger Equation With a Symmetric Parabolic Potential Wall
}

\author{
Soon-Mo Jung ${ }^{1,+}$ iD and Byungbae Kim $^{2, *,+}$ \\ 1 Mathematics Section, College of Science and Technology, Hongik University, Sejong 30016, Korea; \\ smjung@hongik.ac.kr \\ 2 Physics Section, College of Science and Technology, Hongik University, Sejong 30016, Korea \\ * Correspondence: bkim@hongik.ac.kr \\ $\dagger$ These authors contributed equally to this work.
}

Received: 8 June 2020; Accepted: 25 June 2020; Published: 1 July 2020

\begin{abstract}
The first author has recently investigated a type of Hyers-Ulam stability of the one-dimensional time independent Schrödinger equation when the relevant system has a rectangular potential barrier of finite height. In the present paper, we will investigate a type of Hyers-Ulam stability of the Schrödinger equation with the symmetric parabolic wall potential.
\end{abstract}

Keywords: perturbations; Hyers-Ulam stability; Schrödinger equation; time independent Schrödinger equation; symmetric potential wall

MSC: 34D10; 34A40; 34A45; 39B82; 41A30

\section{Introduction}

In 1940, S. M. Ulam [1] discussed a number of important unsolved problems in a mathematics club at the University of Wisconsin. Among them was the following question about the stability of group homomorphisms:

Let $G_{1}$ be a group and let $G_{2}$ be a metric group with the metric $d(\cdot, \cdot)$. Given $\varepsilon>0$, does there exist a

$\delta>0$ such that if a function $h: G_{1} \rightarrow G_{2}$ satisfies the inequality $d(h(x y), h(x) h(y))<\delta$ for all

$x, y \in G_{1}$, then there exists a homomorphism $H: G_{1} \rightarrow G_{2}$ with $d(h(x), H(x))<\varepsilon$ for all $x \in G_{1}$ ?

In 1941, D. H. Hyers [2] solved Ulam's question for approximately additive functions, where $G_{1}$ and $G_{2}$ are Banach spaces. In fact, he demonstrated that we can approximate every solution to $\|f(x+y)-f(x)-f(y)\| \leq \varepsilon$ (for $x, y \in G_{1}$ ) by an additive function. In that case, we say that the Cauchy additive functional equation $f(x+y)=f(x)+f(y)$ has the Hyers-Ulam stability.

Meanwhile, Th. M. Rassias [3] tried to weaken the conditions for the Cauchy differences by attempting not to restrict them strongly:

$$
\|f(x+y)-f(x)-f(y)\| \leq \varepsilon\left(\|x\|^{p}+\|y\|^{p}\right),
$$

where $p<1$ is a fixed real number, and he demonstrated the theorem of Hyers. That is, he proved the Hyers-Ulam-Rassias stability (or the generalized Hyers-Ulam stability) of the Cauchy additive equation. Since then, P. Găvruţa [4] has published a paper that further expanded the theorem of Rassias, both of which have been interesting enough to attract the attention of many mathematicians (see [5-7]). 
Now we assume that $I=(a, b)$ is an open interval $(-\infty \leq a<b \leq+\infty)$ and $n>0$ is a fixed integer. Let us consider the following $n$ th-order linear differential equation

$$
a_{n}(x) y^{(n)}(x)+a_{n-1}(x) y^{(n-1)}(x)+\cdots+a_{1}(x) y^{\prime}(x)+a_{0}(x) y(x)=g(x),
$$

where the function $y: I \rightarrow \mathbb{C}$ is $n$ times continuously differentiable, the coefficient functions $a_{0}, \ldots, a_{n}$ : $I \rightarrow \mathbb{C}$ are continuous, and the function $g: I \rightarrow \mathbb{C}$ is continuous.

We say that if the following statement is true for all $\varepsilon>0$, the differential Equation (1) has Hyers-Ulam stability: If an $n$ times continuously differentiable function $y: I \rightarrow \mathbb{C}$ satisfies

$$
\left|a_{n}(x) y^{(n)}(x)+a_{n-1}(x) y^{(n-1)}(x)+\cdots+a_{1}(x) y^{\prime}(x)+a_{0}(x) y(x)-g(x)\right| \leq \varepsilon
$$

for any $x \in I$, then there is a solution $y_{0}: I \rightarrow \mathbb{C}$ to (1) such that

$$
\left|y(x)-y_{0}(x)\right| \leq K(x, \varepsilon)
$$

for each $x \in I$, where $K(x, \varepsilon)$ depends on $x$ and $\varepsilon$ and where $\lim _{\varepsilon \rightarrow 0} K(x, \varepsilon)=0$ for any value of $x$.

If the limit $\lim _{\varepsilon \rightarrow 0} K(x, \varepsilon)$ indeed depends on the value of $x$, it appears to be somewhat suitable for Hyers-Ulam-Rassias stability in a broad sense, but not in its strict sense. Therefore, the differential Equation (1) may be said to have a type of Hyers-Ulam stability because there is no appropriate official terminology yet. For a more detailed and accurate definition of Hyers-Ulam stability, see [5-7].

As far as we know, M. Obłoza [8,9] is the first mathematician to demonstrate Hyers-Ulam stability of differential equations. Indeed, Obłoza has fully demonstrated the Hyers-Ulam stability of the linear differential equation:

$$
y^{\prime}(x)+f(x) y(x)=g(x)
$$

Since then, many mathematicians have dealt with this topic more broadly and in depth (see [3,10-20]). In a recent paper [21], the first author, together with Choi, explored a type of Hyers-Ulam stability for the one-dimensional time independent Schrödinger equation

$$
-\frac{\hbar^{2}}{2 m} \frac{d^{2} \psi(x)}{d x^{2}}+V(x) \psi(x)=E \psi(x)
$$

when the system under observation has a rectangular potential barrier of finite height.

In the present paper, we will prove a type of Hyers-Ulam stability of the one-dimensional time-independent Schrödinger Equation (3) with the symmetric parabolic wall potential, where $\psi: \mathbb{R} \rightarrow \mathbb{C}$ is the wave function, $V$ is a symmetric parabolic potential function, $\hbar$ is the reduced Planck constant, $m$ is the mass of the particle, and $E$ is the energy of the particle with $E>0$.

Finally, we need to mention that we are writing this paper using the ideas and experience of the papers $[16,18,21,22]$.

\section{Preliminaries}

The formula expressing the solution to the first-order linear inhomogeneous differential Equation (2) is widely known. In the next lemma, we want to recall this theorem once again.

Lemma 1. Suppose that the functions $f, g: \mathbb{R} \rightarrow \mathbb{C}$ are continuous and each of the integrals below exists. Every continuously differentiable function $y: \mathbb{R} \rightarrow \mathbb{C}$ is a solution of the first-order linear inhomogeneous differential Equation (2) if and only if $y$ can be expressed as

$$
y(x)=\exp \left(-\int_{0}^{x} f(w) d w\right)\left(y(c)+\int_{0}^{x} g(s) \exp \left(\int_{0}^{s} f(w) d w\right) d s\right)
$$


where $y(c)$ is an arbitrary complex number.

By using Lemma 1, we can easily prove the generalized Hyers-Ulam stability (or the Hyers-Ulam-Rassias stability) of the first-order linear inhomogeneous differential Equation (2). Obłoza proved the Hyers-Ulam stability version of the following theorem long ago, but for the convenience of our readers, we want to prove it again here in a way that is a little different from the Obłoza's way. As usual, we denote by $\Re(z)$ the real part of any complex number $z$.

Lemma 2. Suppose that the functions $f, g: \mathbb{R} \rightarrow \mathbb{C}$ and $\varphi: \mathbb{R} \rightarrow[0, \infty)$ are continuous and each of the integrals below exists. If a continuously differentiable function $y: \mathbb{R} \rightarrow \mathbb{C}$ satisfies

$$
\left|y^{\prime}(x)+f(x) y(x)-g(x)\right| \leq \varphi(x)
$$

for any $x \in \mathbb{R}$, then there is a continuously differentiable solution $y_{0}: \mathbb{R} \rightarrow \mathbb{C}$ to the first-order linear inhomogeneous differential Equation (2) such that

$$
\left|y(x)-y_{0}(x)\right| \leq \exp \left(-\Re\left(\int_{0}^{x} f(w) d w\right)\right)\left|\int_{0}^{x} \varphi(s) \exp \left(\Re\left(\int_{0}^{s} f(w) d w\right)\right) d s\right|
$$

for all $x \in \mathbb{R}$.

Proof. First, we define $h(x)=y^{\prime}(x)+f(x) y(x)-g(x)$ for each $x \in \mathbb{R}$. Obviously, the function $h: \mathbb{R} \rightarrow \mathbb{C}$ is continuous and $|h(x)| \leq \varphi(x)$ for any $x \in \mathbb{R}$. On account of Lemma 1 , we get

$$
y(x)=\exp \left(-\int_{0}^{x} f(w) d w\right)\left(y(c)+\int_{0}^{x}(g(s)+h(s)) \exp \left(\int_{0}^{s} f(w) d w\right) d s\right),
$$

where $y(c)$ is an arbitrary complex number.

Now we set

$$
y_{0}(x)=\exp \left(-\int_{0}^{x} f(w) d w\right)\left(y(c)+\int_{0}^{x} g(s) \exp \left(\int_{0}^{s} f(w) d w\right) d s\right)
$$

for any $x \in \mathbb{R}$. Then, the function $y_{0}: \mathbb{R} \rightarrow \mathbb{C}$ is continuously differentiable and it is a solution to the first-order linear differential Equation (2) by Lemma 1. Furthermore, we have

$$
\begin{aligned}
\left|y(x)-y_{0}(x)\right| & =\left|\exp \left(-\int_{0}^{x} f(w) d w\right) \int_{0}^{x} h(s) \exp \left(\int_{0}^{s} f(w) d w\right) d s\right| \\
& \leq\left|\exp \left(-\int_{0}^{x} f(w) d w\right)\right| \cdot\left|\int_{0}^{x}\right| h(s)|| \exp \left(\int_{0}^{s} f(w) d w\right)|d s| \\
& \leq \exp \left(-\Re\left(\int_{0}^{x} f(w) d w\right)\right)\left|\int_{0}^{x} \varphi(s) \exp \left(\Re\left(\int_{0}^{s} f(w) d w\right)\right) d s\right|
\end{aligned}
$$

for each $x \in \mathbb{R}$.

\section{A Type of Hyers-Ulam Stability}

In this section, suppose that the potential function $V: \mathbb{R} \rightarrow \mathbb{R}$ is a parabolic function defined by

$$
\begin{aligned}
V(x) & =\frac{\hbar^{2} \alpha^{2}}{2 m} x^{2}-\frac{\hbar^{2} \alpha \beta}{m} x+\frac{\hbar^{2}}{2 m}\left(\alpha+\beta^{2}\right)+E \\
& =\frac{\hbar^{2}}{2 m}(\alpha x-\beta)^{2}+\frac{\hbar^{2}}{2 m} \alpha+E,
\end{aligned}
$$


where $\alpha, \beta$ are real constants and $E>0$. We will focus on the symmetric parabolic potential function with $\alpha<0$ in the last part of this section.

Considering the form of the potential function $V$ given in (4), we define the differential operators $\mathcal{L}_{1}$ and $\mathcal{L}_{2}$ as follows:

$$
\begin{aligned}
& \left(\mathcal{L}_{1} \psi\right)(x)=\psi^{\prime}(x)+(\beta-\alpha x) \psi(x), \\
& \left(\mathcal{L}_{2} \psi\right)(x)=\psi^{\prime}(x)+(\alpha x-\beta) \psi(x)
\end{aligned}
$$

for all continuously differentiable functions $\psi: \mathbb{R} \rightarrow \mathbb{C}$.

For all twice continuously differentiable functions $\psi: \mathbb{R} \rightarrow \mathbb{C}$, it follows from (4) and (5) that

$$
\begin{aligned}
-\frac{\hbar^{2}}{2 m}\left(\left(\mathcal{L}_{2} \circ \mathcal{L}_{1}\right) \psi\right)(x) & =-\frac{\hbar^{2}}{2 m}\left(\psi^{\prime \prime}(x)+\left(-\alpha^{2} x^{2}+2 \alpha \beta x-\beta^{2}-\alpha\right) \psi(x)\right) \\
& =-\frac{\hbar^{2}}{2 m} \psi^{\prime \prime}(x)+V(x) \psi(x)-E \psi(x)
\end{aligned}
$$

for each $x \in \mathbb{R}$, which means that

$$
\left|-\frac{\hbar^{2}}{2 m} \psi^{\prime \prime}(x)+V(x) \psi(x)-E \psi(x)\right| \leq \varepsilon \quad(x \in \mathbb{R})
$$

if and only if

$$
\left|\left(\left(\mathcal{L}_{2} \circ \mathcal{L}_{1}\right) \psi\right)(x)\right| \leq \frac{2 m}{\hbar^{2}} \varepsilon \quad(x \in \mathbb{R})
$$

which is again equivalent to

$$
\left|\phi^{\prime}(x)+(\alpha x-\beta) \phi(x)\right| \leq \frac{2 m}{\hbar^{2}} \varepsilon \quad(x \in \mathbb{R}),
$$

where we set $\phi(x)=\left(\mathcal{L}_{1} \psi\right)(x)$.

Since the inequality (8) below is strongly affected by the value of $x$, the phenomenon observed in the following theorem is called a type of Hyers-Ulam stability.

Theorem 1. Assume that $V: \mathbb{R} \rightarrow \mathbb{R}$ is the parabolic potential function defined by (4), where $\alpha, \beta$ are real constants and $E>0$ is a real number as the energy of the particle under observation. Given any $\varepsilon>0$, if a twice continuously differentiable function $\psi: \mathbb{R} \rightarrow \mathbb{C}$ satisfies

$$
\left|-\frac{\hbar^{2}}{2 m} \frac{d^{2} \psi(x)}{d x^{2}}+V(x) \psi(x)-E \psi(x)\right| \leq \varepsilon
$$

for any $x \in \mathbb{R}$, then there is a twice continuously differentiable solution $\psi_{0}: \mathbb{R} \rightarrow \mathbb{C}$ of the one-dimensional time independent Schrödinger Equation (3) such that

$$
\begin{aligned}
& \left|\psi(x)-\psi_{0}(x)\right| \\
& \leq \frac{2 m}{\hbar^{2}} \varepsilon \exp \left(\frac{\alpha}{2} x^{2}-\beta x\right)\left|\int_{0}^{x} \exp \left(-\alpha s^{2}+2 \beta s\right)\right| \int_{0}^{s} \exp \left(\frac{\alpha}{2} w^{2}-\beta w\right) d w|d s|
\end{aligned}
$$

for every $x \in \mathbb{R}$.

Proof. We set $\phi(x)=\left(\mathcal{L}_{1} \psi\right)(x)=\psi^{\prime}(x)+(\beta-\alpha x) \psi(x)$. On account of (6) and (7), we may apply Lemma 2 to inequality (6), referring to the substitutions shown in the following Table 1 : 
Table 1. Substitutions.

\begin{tabular}{cc}
\hline Lemma 2 & in $(6)$ \\
\hline$y(x)$ & $\phi(x)$ \\
$f(x)$ & $\alpha x-\beta$ \\
$g(x)$ & 0 \\
$\varphi(x)$ & $\frac{2 m}{\hbar^{2} \varepsilon}$ \\
\hline
\end{tabular}

We conclude that there is a continuously differentiable function $\phi_{0}: \mathbb{R} \rightarrow \mathbb{C}$ such that

$$
\phi_{0}^{\prime}(x)+(\alpha x-\beta) \phi_{0}(x)=0
$$

and

$$
\begin{aligned}
\left|\phi(x)-\phi_{0}(x)\right| & \leq \frac{2 m}{\hbar^{2}} \varepsilon \exp \left(-\int_{0}^{x}(\alpha w-\beta) d w\right)\left|\int_{0}^{x} \exp \left(\int_{0}^{s}(\alpha w-\beta) d w\right) d s\right| \\
& =\frac{2 m}{\hbar^{2}} \varepsilon\left|\int_{0}^{x} \exp \left(\beta(x-s)-\frac{\alpha}{2}\left(x^{2}-s^{2}\right)\right) d s\right|
\end{aligned}
$$

for every $x \in \mathbb{R}$.

Since $\phi(x)=\psi^{\prime}(x)+(\beta-\alpha x) \psi(x)$, it follows from (10) that

$$
\left|\psi^{\prime}(x)+(\beta-\alpha x) \psi(x)-\phi_{0}(x)\right| \leq \frac{2 m}{\hbar^{2}} \varepsilon\left|\int_{0}^{x} \exp \left(\beta(x-s)-\frac{\alpha}{2}\left(x^{2}-s^{2}\right)\right) d s\right|
$$

for all $x \in \mathbb{R}$. We can apply Lemma 2 to the inequality (11) with reference to the substitutions presented in the following Table 2:

Table 2. Substitutions.

\begin{tabular}{cc}
\hline Lemma 2 & in $(11)$ \\
\hline$y(x)$ & $\psi(x)$ \\
$f(x)$ & $\beta-\alpha x$ \\
$g(x)$ & $\phi_{0}(x)$ \\
$\varphi(x)$ & $\frac{2 m}{\hbar^{2}} \varepsilon\left|\int_{0}^{x} \exp \left(\beta(x-s)-\frac{\alpha}{2}\left(x^{2}-s^{2}\right)\right) d s\right|$ \\
\hline
\end{tabular}

In view of Lemma 2, there is a continuously differentiable function $\psi_{0}: \mathbb{R} \rightarrow \mathbb{C}$ that satisfies

$$
\psi_{0}^{\prime}(x)+(\beta-\alpha x) \psi_{0}(x)=\phi_{0}(x)
$$

and

$$
\left|\psi(x)-\psi_{0}(x)\right| \leq \frac{2 m}{\hbar^{2}} \varepsilon \exp \left(\frac{\alpha}{2} x^{2}-\beta x\right)\left|\int_{0}^{x} \exp \left(-\alpha s^{2}+2 \beta s\right)\right| \int_{0}^{s} \exp \left(\frac{\alpha}{2} w^{2}-\beta w\right) d w|d s|
$$

for all $x \in \mathbb{R}$.

Moreover, by Lemma 1 and (12), the $\psi_{0}$ has the form

$$
\psi_{0}(x)=\exp \left(\frac{\alpha}{2} x^{2}-\beta x\right)\left(\psi_{0}(c)+\int_{0}^{x} \phi_{0}(s) \exp \left(-\frac{\alpha}{2} s^{2}+\beta s\right) d s\right),
$$

where $\psi_{0}(c)$ is an arbitrary complex number. Since $\phi_{0}$ is continuously differentiable, we see that the $\psi_{0}$ is twice continuously differentiable.

Finally, by making use of (9) and (12), it is easy to show that $\psi_{0}: \mathbb{R} \rightarrow \mathbb{C}$ is a solution to the one-dimensional time independent Schrödinger Equation (3). 
For each real constants $\alpha$ and $\beta$ with $\alpha<0$, we know that

$$
\begin{aligned}
\left|\int_{0}^{s} \exp \left(\frac{\alpha}{2} w^{2}-\beta w\right) d w\right| & =\exp \left(-\frac{\beta^{2}}{2 \alpha}\right)\left|\int_{-\beta / \alpha}^{s-\beta / \alpha} \exp \left(\frac{\alpha}{2} u^{2}\right) d u\right| \\
& \leq \exp \left(-\frac{\beta^{2}}{2 \alpha}\right) \int_{-\infty}^{\infty} \exp \left(\frac{\alpha}{2} u^{2}\right) d u \\
& =\sqrt{-\frac{2 \pi}{\alpha}} \exp \left(-\frac{\beta^{2}}{2 \alpha}\right)
\end{aligned}
$$

for all $s \in \mathbb{R}$.

Hence, it follows from (8) that

$$
\begin{aligned}
\left|\psi(x)-\psi_{0}(x)\right| & \leq \frac{2 m}{\hbar^{2}} \sqrt{-\frac{2 \pi}{\alpha}} \varepsilon \exp \left(\frac{\alpha}{2} x^{2}-\beta x-\frac{\beta^{2}}{2 \alpha}\right)\left|\int_{0}^{x} \exp \left(-\alpha s^{2}+2 \beta s\right) d s\right| \\
& =\frac{2 m}{\hbar^{2}} \sqrt{-\frac{2 \pi}{\alpha}} \varepsilon \exp \left(\frac{\alpha}{2} x^{2}-\beta x+\frac{\beta^{2}}{2 \alpha}\right)\left|\int_{0}^{x} \exp \left(-\alpha\left(s-\frac{\beta}{\alpha}\right)^{2}\right) d s\right|
\end{aligned}
$$

for all $x \in \mathbb{R}$.

If we put $\alpha<0$ and $\beta=0$ in (4) and (13), then we see the corollary.

Corollary 1. Suppose $V: \mathbb{R} \rightarrow \mathbb{R}$ is the symmetric parabolic potential function defined by

$$
V(x)=\frac{\hbar^{2} \alpha^{2}}{2 m} x^{2}+\frac{\hbar^{2} \alpha}{2 m}+E,
$$

where $\alpha<0$ is a real number and $E>0$ is a real number as the energy of the particle under observation. Given any $\varepsilon>0$, if a twice continuously differentiable function $\psi: \mathbb{R} \rightarrow \mathbb{C}$ satisfies (7) for any $x \in \mathbb{R}$, then there is a twice continuously differentiable solution $\psi_{0}: \mathbb{R} \rightarrow \mathbb{C}$ to the one-dimensional time independent Schrödinger Equation (3) with

$$
\left|\psi(x)-\psi_{0}(x)\right| \leq \frac{2 m}{\hbar^{2}} \sqrt{-\frac{2 \pi}{\alpha}} \varepsilon \exp \left(\frac{\alpha}{2} x^{2}\right)\left|\int_{0}^{x} \exp \left(-\alpha s^{2}\right) d s\right|
$$

for all $x \in \mathbb{R}$.

In addition, if we set $\alpha=-\frac{2 m E}{\hbar^{2}}$ in formula (14) for the symmetric potential function, then we have

$$
V(x)=\frac{2 m E^{2}}{\hbar^{2}} x^{2}
$$

and the corresponding Schrödinger equation is closely related to the quantum-mechanical harmonic oscillator.

Corollary 2. Suppose $V: \mathbb{R} \rightarrow \mathbb{R}$ is the symmetric parabolic potential function defined by (15). Given any $\varepsilon>0$, if a twice continuously differentiable function $\psi: \mathbb{R} \rightarrow \mathbb{C}$ satisfies (7) for any $x \in \mathbb{R}$, then there is a twice continuously differentiable solution $\psi_{0}: \mathbb{R} \rightarrow \mathbb{C}$ to the one-dimensional time independent Schrödinger Equation (3) with

$$
\left|\psi(x)-\psi_{0}(x)\right| \leq \frac{2}{\hbar} \sqrt{\frac{m \pi}{E}} \varepsilon \exp \left(-\frac{m E}{\hbar^{2}} x^{2}\right)\left|\int_{0}^{x} \exp \left(\frac{2 m E}{\hbar^{2}} s^{2}\right) d s\right|
$$

for every $x \in \mathbb{R}$. In particular, the $\psi_{0}$ is given by (17). 
Proof. Assume that $\psi_{0}: \mathbb{R} \rightarrow \mathbb{C}$ is a solution of the one-dimensional time independent Schrödinger Equation (3) with the potential function given in (15). It only needs to prove that the $\psi_{0}$ is given by (17) below.

We set $\xi=\frac{\sqrt{2 m E}}{\hbar} x$ for all $x \in \mathbb{R}$. Then $\xi \in \mathbb{R}$ if and only if $x \in \mathbb{R}$. Moreover, we put

$$
\phi_{0}(\xi)=\psi_{0}\left(\frac{\hbar}{\sqrt{2 m E}} \xi\right)=\psi_{0}(x)
$$

for all $\xi \in \mathbb{R}$. Then we have

$$
-\frac{\hbar^{2}}{2 m} \frac{d^{2} \psi_{0}(x)}{d x^{2}}=-\frac{\hbar^{2}}{2 m} \frac{2 m E}{\hbar^{2}} \frac{d^{2} \phi_{0}(\xi)}{d \xi^{2}}=-E \frac{d^{2} \phi_{0}(\xi)}{d \xi^{2}} .
$$

Hence, if we assume that the potential function $V$ is given by (15), then we get

$$
-\frac{\hbar^{2}}{2 m} \frac{d^{2} \psi_{0}(x)}{d x^{2}}+V(x) \psi_{0}(x)-E \psi_{0}(x)=-E\left(\frac{d^{2} \phi_{0}(\xi)}{d \xi^{2}}+\left(1-\xi^{2}\right) \phi_{0}(\xi)\right)=0
$$

for all $\xi \in \mathbb{R}$.

Furthermore, it is obvious that $\phi_{0}$ is given as

$$
\phi_{0}(\xi)=\exp \left(-\frac{\xi^{2}}{2}\right)\left(C_{1}+C_{2} \int_{0}^{\xi} e^{w^{2}} d w\right)
$$

where $C_{1}$ and $C_{2}$ are fixed complex constants. By (16), the last expression for $\phi_{0}$ is equivalent to

$$
\psi_{0}(x)=\exp \left(-\frac{m E}{\hbar^{2}} x^{2}\right)\left(C_{1}+C_{2} \int_{0}^{x} \exp \left(\frac{2 m E}{\hbar^{2}} w^{2}\right) d w\right),
$$

and we may show that the $\psi_{0}$ is a particular solution of the one-dimensional time independent Schrödinger equation (3) with the symmetric potential function $V$ given in (15).

\section{Discussion}

The Schrödinger equation is based on the postulates of quantum mechanics and this method can be applied to the perturbation theory when it is very hard to find the exact solution for some potentials. It is also possible to apply the one-dimensional Schrödinger equation to analyze the state of the particles reflected by the rectangular potential, which is less relevant to the subject matter of this paper.

Because the difference between the perturbed solution $\psi$ and the exact solution $\psi_{0}$ to the one-dimensional time independent Schrödinger Equation (3) is influenced by $x$, we have not proved in Theorem 1, Corollaries 1 and 2, the exact Hyers-Ulam stability of that Schrödinger equation when the potential is a (symmetric) parabolic wall and $E>0$. Therefore we can say that we have proved a type of Hyers-Ulam stability in this paper.

It is necessary to improve Lemma 2 in order to demonstrate the exact Hyers-Ulam stability of the Schrödinger Equation (3) with symmetric parabolic wall potential, but we think it may take quite a while to accomplish this and we want to leave this improvement as an open problem.

\section{Conclusions}

In the present paper, we have proved a type of Hyers-Ulam stability of one-dimensional time-independent Schrödinger equation by applying the operator method when the potential function is expressed in the form of (4). Our main result does not cover the more general case of Hyers-Ulam stability of the Schrödinger equation when the potential function is $V(x)=\alpha x^{2}+\beta x+\gamma$, where parameters $\alpha, \beta$, and $\gamma$ meet the minimum necessary conditions. Nevertheless, it is impressive 
and worthwhile that we showed a type of Hyers-Ulam stability of the Schrödinger equation with the (symmetric) potential function of a smooth curve shape.

Author Contributions: Conceptualization, S.-M.J. and B.K.; writing-original draft preparation, S.-M.J. and B.K.; writing-review and editing, S.-M.J. and B.K.; funding acquisition, B.K. All authors have read and agreed to the published version of the manuscript.

Funding: This work was supported by 2018 Hongik University Research Fund.

Conflicts of Interest: The authors declare no conflict of interest.

\section{References}

1. Ulam, S.M. A Collection of Mathematical Problems; Interscience Publ.: New York, NY, USA, 1960.

2. Hyers, D.H. On the stability of the linear functional equation. Proc. Natl. Acad. Sci. USA 1941, 27, 222-224. [CrossRef] [PubMed]

3. Rassias, T.M. On the stability of the linear mapping in Banach spaces. Proc. Am. Math. Soc. 1978, 72, 297-300. [CrossRef]

4. Găvruța, P. A generalization of the Hyers-Ulam-Rassias stability of approximately additive mappings. J. Math. Anal. Appl. 1994, 184, 431-436. [CrossRef]

5. Hyers, D.H.; Isac, G.; Rassias, T.M. Stability of Functional Equations in Several Variables; Birkhäuser: Boston, MA, USA, 1998.

6. Jung, S.-M. Hyers-Ulam-Rassias Stability of Functional Equations in Nonlinear Analysis; Springer: New York, NY, USA, 2011.

7. Sahoo, P.K.; Kannappan, P.L. Introduction to Functional Equations; CRC Press: Boca Raton, FL, USA, 2011.

8. Obłoza, M. Hyers stability of the linear differential equation. Rocznik Nauk.-Dydakt. Prace Mat. 1993, 13, 259-270.

9. Obłoza, M. Connections between Hyers and Lyapunov stability of the ordinary differential equations. Rocznik Nauk.-Dydakt. Prace Mat. 1997, 14, 141-146.

10. Alsina, C.; Ger, R. On some inequalities and stability results related to the exponential function. J. Inequal. Appl. 1998, 2, 373-380. [CrossRef]

11. Choi, G.; Jung, S.-M. Invariance of Hyers-Ulam stability of linear differential equations and its applications. Adv. Difference Equ. 2015, 277, 1-14. [CrossRef]

12. Choi, G.; Jung, S.-M.; Roh, J. Some properties of approximate solutions of linear differential equations. Mathematics 2019, 7, 806. [CrossRef]

13. Cîmpean, D.S.; Popa, D. On the stability of the linear differentia equation of higher order with constant coefficients. Appl. Math. Comput. 2010, 217, 4141-4146.

14. Jung, S.-M. Hyers-Ulam stability of linear differential equations of first order. Appl. Math. Lett. 2004, 17, 1135-1140. [CrossRef]

15. Jung, S.-M. Hyers-Ulam stability of linear differential equations of first order, II. Appl. Math. Lett. 2006, 19, 854-858. [CrossRef]

16. Jung, S.-M.; Roh, J. Hyers-Ulam stability of the time independent Schrödinger equations. Appl. Math. Lett. 2017, 74, 147-153. [CrossRef]

17. Li, Y.; Shen, Y. Hyers-Ulam stability of linear differential equations of second order. Appl. Math. Lett. 2010, 23, 306-309. [CrossRef]

18. Popa, D.; Raşa, I. On the Hyers-Ulam stability of the linear differential equation. J. Math. Anal. Appl. 2011, 381, 530-537. [CrossRef]

19. Takahasi, S.-E.; Miura, T.; Miyajima, S. On the Hyers-Ulam stability of the Banach space-valued differential equation $y^{\prime}=\lambda y$. Bull. Korean Math. Soc. 2002, 39, 309-315. [CrossRef]

20. Wang, G.; Zhou, M.; Sun, L. Hyers-Ulam stability of linear differential equations of first order. Appl. Math. Lett. 2008, 21, 1024-1028. [CrossRef] 
21. Choi, G.; Jung, S.-M. Perturbation of one-dimensional time independent Schrödinger equation with a rectangular potential barrier. under review.

22. Choi, G.; Jung, S.-M.; Roh, J. An operator method for the stability of inhomogeneous wave equations. Symmetry 2019, 11, 324. [CrossRef]

(C) 2020 by the authors. Licensee MDPI, Basel, Switzerland. This article is an open access article distributed under the terms and conditions of the Creative Commons Attribution (CC BY) license (http:/ / creativecommons.org/licenses/by/4.0/). 